\title{
Quand les Taviani réécrivent Shakespeare : Cesare deve morire (2012)
}

\section{Erik Pesenti Rossi}

\section{(2) OpenEdition}

1 Journals

Édition électronique

URL : https://journals.openedition.org/cher/2179

DOI : 10.4000/cher.2179

ISSN : 2803-5992

Éditeur

Presses universitaires de Strasbourg

\section{Édition imprimée}

Date de publication : 29 mai 2018

Pagination : 79-86

ISBN : 979-10-344-0019-5

ISSN : 1968-035X

\section{Référence électronique}

Erik Pesenti Rossi, «Quand les Taviani réécrivent Shakespeare : Cesare deve morire (2012) »,

reCHERches [En ligne], 20 | 2018, mis en ligne le 01 décembre 2021, consulté le 02 décembre 2021.

URL : http://journals.openedition.org/cher/2179; DOI : https://doi.org/10.4000/cher.2179

\section{(c) (i) (8)}

Ce(tte) œuvre est mise à disposition selon les termes de la Licence Creative Commons Attribution Pas d'Utilisation Commerciale - Partage dans les Mêmes Conditions 4.0 International. 


\title{
Quand les Taviani réécrivent Shakespeare: Cesare deve morire (2012)
}

\author{
ERIK PESENTI ROSSI ${ }^{1}$
}

\begin{abstract}
$\mathrm{E}$ n 2011, les cinéastes Paolo et Vittorio Taviani sont contactés par une amie attachée de presse qui s'occupe du théâtre de la prison de Rebibbia à Rome, pour venir assister à une des représentations montées par les prisonniers ${ }^{2}$. Émus par ce spectacle, ils décident à leur tour d'écrire un film (tourné en 21 jours) qui raconte la mise en scène de Jules César de Shakespeare par des prisonniers de Rebibbia. Il s'agit donc bien d'un film, et non point de théâtre filmé; tout au plus peut-on parler de «docufiction». Dans ce travail ils sont aidés par Fabio Cavalli qui dirige la troupe théâtrale de Rebibbia et qui va réécrire les dialogues.
\end{abstract}

Les acteurs sont les prisonniers (condamnés à de lourdes peines); pour les décors, ce sont les différents endroits de la prison (cellules, couloirs, cours de promenade, bibliothèque) ainsi que la salle de spectacle de celle-ci.

Les Taviani choisissent un montage particulier: on commence par montrer la fin de la représentation, puis, six mois plus tôt, dans un flash-back qui va constituer l'essentiel du film, on filme la découverte de la pièce par les prisonniers, puis les répétitions. La vraie réécriture de la pièce est là, dans ce qui se passe dans les "coulisses", où chacun va s'assimiler à son propre rôle, mais aussi (comme on va le voir) retrouver dans sa propre vie des situations analogues à l'histoire de Shakespeare. À la fin du film (en noir et blanc pour les répétitions), la fin du «vrai» spectacle (en couleur) apparaîtra à nouveau.

En ce qui concerne le texte de Shakespeare, il faut d'abord remarquer le changement de titre (César doit mourir), indiquant, d'une part, que l'on n'est pas dans la simple adaptation, mais bien dans un film original; et, d'autre part, qu'un axe particulier de la pièce est choisi : celui de l'assassinat et de la trahison, pour raison politique, par vengeance et calcul personnel, ou pour d'autres

1 Professeur, membre du CHER, Université de Strasbourg.

2 Lorsqu'ils arrivent, il y avait un détenu qui lisait en napolitain l'épisode de «Paolo e Francesca», tiré de l'Enfer de Dante. 
raisons humaines que chaque acteur-détenu peut aisément comprendre; c'est ce qui rend l'intrigue universelle et profondément humaine. La chronologie de l'intrigue est, dans l'ensemble, suivie, mais les Taviani n'en conservent que les moments et les répliques essentielles. Comme on va le voir, les dialogues sont reconstruits: ils mêlent ceux du texte et ceux des acteurs.

$*$

Dans les pages qui suivent, je vais exposer brièvement le découpage du film, tout en le commentant afin de bien mettre en évidence la manière dont on procède à la réécriture de Shakespeare.

Avant la pièce (environ 17 minutes):

- On voit, en couleur, la fin de la représentation de la pièce Jules César (mort de Brutus). Des sous-titres informent que nous sommes à Rome, à la prison de Rebibbia, dans le quartier de haute sécurité.

- Plan sur la salle vide. Les prisonniers se rhabillent et retournent dans leurs cellules.

- Flash-back, six mois avant. À partir d'ici, le film est en noir et blanc. Fabio Cavalli présente le projet aux détenus.

- Les acteurs font des essais. Ils doivent se présenter de deux manières différentes (triste et enragée) dans une même situation.

- Distribution des rôles par le metteur en scène. On en profite pour les présenter: Giovanni Arcuri (César), condamné à 17 ans de prison pour trafic de stupéfiants; Salvatore Striano - dit Sasà - (Brutus), condamné à 14 ans et 8 mois pour association mafieuse; certains sont condamnés à perpétuité.

- On commence à s'entraîner à jouer, assis dans le noir, en rond dans une salle. Les acteurs ont lu leur rôle, mais il faut commencer à le dire. On commence par l'acte I, scène I. Si chacun joue dans son propre dialecte (sicilien, romain, etc.), il faut essayer de parler dans une langue pas trop populaire; un des détenus n'a pas de dialecte et se déclare «citoyen du monde», mais il peut parler en anglais ou en néerlandais. Chaque détenu ajoute son commentaire et tend à choisir son rôle tout en l'adaptant à sa vision des choses; ainsi celui qui interprète le devin, dit que chez lui les magiciens sont tous un peu fous, et que donc il va jouer le rôle d'un fou.

Acte $\mathrm{I}^{3}$ :

- Cosimo Rega (Cassius) assis sur son lit, dans sa cellule, relit son rôle. Sasà (Brutus) fait de même, dans sa propre cellule, mais il marche dans sa cellule. Tous deux essaient de s'imprégner du personnage qu'ils doivent interpréter. Ils semblent se répondre d'une cellule à l'autre, et, de ce fait, ils sont montrés en alternance. On reprend ici le dialogue entre Brutus et

3 J'indique les actes et les scènes identifiables dans le film; de nombreuses scènes sont éliminées ou fondues avec d'autres par les Taviani. 
Cassius de la scène II, en l'adaptant et en le résumant (comme on le fera dans presque tout le film).

- Brutus et Cassius, dans un couloir de la prison. Ils entendent du bruit au dehors dans la cour : cela pourrait être la foule de Rome au temps de César; immédiatement ils enchaînent sur leur rôle: nous sommes au moment où César se présente au peuple et refuse trois fois la couronne. Dans le texte, la scène ${ }^{4}$ est d'abord vue par Brutus («Une autre acclamation! Je crois qu'on applaudit à de nouveaux honneurs qui accablent César ${ }^{5}$.») puis racontée par Casca; ici elle est décrite par Cassius depuis une fenêtre du couloir. Cassius (Cosimo) déclare «Roma, città senza vergogna» et enchaîne sur sa propre ville, Naples, devenue elle aussi une "ville sans vergogne». Par cette réplique personnelle ${ }^{6}$ on voit que l'acteur s'assimile au personnage. Il s'excuse de cette digression personnelle et dit qu'il a vraiment l'impression que Shakespeare a vécu à Naples. César (Giovanni Arcuri) passe avec Antoine. César (Giovanni) fait changer Antoine de côté car «de cette oreille je n'entends vraiment rien $^{7}$ » (ici c'est bien l'acteur qui a un problème d'ouïe, l'humour n'est donc jamais absent dans ce film).

- Dans le théâtre en travaux, rien n'est prêt. On comprend bien que l'essentiel ne semble pas être là.

- Brutus et Lucius dans leur cellule sont en train de répéter. Lucius est allongé, les yeux fermés. Sasà (Brutus): "J’ai compris ce que Shakespeare veut dire, mais il faut que le public comprenne aussi.»

- Sasà répète son rôle dans différents couloirs de la prison. Les couloirs remplacent aisément les rues de Rome, une nuit d'orage.

Acte II (le complot):

- À partir de cette séquence, il devient évident que la «vraie» pièce (et donc le vrai drame du film) est la répétition qui a pour décors les différents endroits de la prison. On commence dans la cellule de Brutus. Nous sommes (idéalement) aux Ides de Mars. Les prisonniers viennent le trouver dans sa prison, comme les conjurés de Shakespeare viennent trouver Brutus dans son palais. Juste avant l'arrivée de Cassius, on voit Brutus seul dans sa cellule et on entend du tonnerre au-dehors (voir Acte I, scène III : «Rome. Une rue. Il fait nuit. Tonnerre et éclairs. ») Pendant que Cassius et Brutus discutent dans la cellule, les autres conjurés font de même dans le couloir : ils disent que l'orage est passé; certains se moquent de Shakespeare, d'autres disent qu'il a raison, que tout est important et

4 Toujours dans la scène II.

5 J'utilise la traduction de François-Victor Hugo.

6 Dans la scène II Cassius déclare: «Siècle tu es dans la honte! Rome, tu as perdu la race des nobles cœurs!» «Rome, ville sans vergogne» n'apparait pas dans le texte de Shakespeare.

7 Pour les traductions de l'italien je reprends les sous-titres du DVD. 
qu'ils n'ont aucun mal à s'assimiler au rôle. Ils jouent, en la commentant, la scène I de l'acte II: «-Décius: C’est ici le levant. N'est-ce pas le jour qui apparaît ici? -Casca: Non. -Cinna: Oh! pardon, monsieur, c'est lui ; et ces lignes grises qui rayent les nuages là-haut, sont les messagères du jour.» Au moment où il dit qu'il ne faut pas tuer Antoine, Sasà n'arrive plus à jouer son rôle de Brutus car, selon lui, la justice ce n'est pas cela. Il sait son rôle, mais il est trop dur à jouer, car trop vrai. «Nous avons tous connu des gens aussi tyranniques que César» dit Cassius, et il est clair qu'il parle de «la vraie vie». En fait ces rôles sont durs car, eux aussi, ont tous connu les vengeances et les assassinats qui y sont liés. Sasà s'énerve avec le metteur en scène: "C'est une chose à moi! C'est une chose à $\mathrm{moi}^{8}$ ! crie-t-il. Il évoque alors un ami à lui qui a dû tuer «un infâme ${ }^{9}$ » et dit les mêmes mots que Brutus: "Oh! si nous pouvions atteindre l'esprit de César, sans déchirer César ${ }^{10}$ !» Sasà continue: tout le monde s'est moqué de cet ami, même lui. On comprend que l'histoire s'est mal terminée. Cette longue séquence (environ sept minutes) se clôt sur la réplique de Brutus qui voit Lucius dormant: «Lucius! enfant!... il dort profondément ${ }^{11}$ !... » mais qui est synthétisée de cette façon: «Eh, toi! Dors. Dors Lucius. C’est mieux comme ça!» (dite en dialecte napolitain).

- Transition avec un poster représentant la mer (rêves de prisonniers), sur un fond de musique légèrement dramatique qui accompagne tout le film.

- Dans la bibliothèque de la prison, les prisonniers discutent. Giovanni admire César (son rôle). Il l'a étudié au lycée. On enchaîne sur l'acte II, scène II (dans le palais de César, César et Décius). À la fin de la scène, Giovanni sort du script et donne son avis sur Décius: ce n'est pas un ami, mais un lèche-bottes. À ce moment-là, Giovanni commence à régler ses comptes personnels avec Juan (Décius): ils vont se battre dans le couloir et risquent de faire annuler la pièce. Cependant, les choses se calment.

- Plan d'ensemble et travellings sur la prison. Plan sur les prisonniers dans les cellules, chacun pense à ses problèmes. C'est la nuit, ils sont dans leurs lits.

\section{Acte III (mort de César)}

- Le lendemain, les prisonniers sortent pour la promenade. César sort.

- Dans la cour de la prison, on joue la scène I de l'acte III, la rencontre entre César et Métellus. César refuse la grâce du frère de Métellus. César est assassiné. Les prisonniers fuient, comme le peuple de Rome dans la pièce. On voit ensuite les comploteurs devant le corps de César. Brutus, changeant un peu la réplique de Shakespeare (qui semble avoir prévu les propres réécritures de son texte): «Combien de fois César devra mourir

8 «É una cosa mia! É una cosa mia!»

9 C'est-à-dire un traître.

10 Acte II, scène I.

11 Acte II, scène I. 
sur une scène de théâtre, comme ici, aujourd'hui, dans notre prison ${ }^{12}$ ». Antoine arrive, qui demande la mort pour lui-même. Il demande ensuite de pouvoir porter le corps au forum. Les gardiens de la prison s'impatientent depuis leur mirador; mais leur chef veut que l'on finisse la scène. Les gardiens, faisant alors comme des spectateurs depuis un hypothétique balcon, se mettent à donner leurs avis sur les personnages. Il y en a un qui aime bien Antoine, car il le trouve "accommodant», un autre dit que c'est «un fils de pute». Ils se prennent au jeu et veulent voir si Antoine est vraiment accommodant. Antoine parle à César. Il jure vengeance (fin de l'acte III, scène II).

- Toujours acte III, scène II. Le corps de César est déposé au centre de la cour de la prison. Les prisonniers, excités, depuis leurs fenêtres, jouent le rôle du peuple de Rome. Brutus s'explique; il propose de se suicider. Le peuple, depuis les fenêtres des cellules, refuse. Arrivée d'Antoine. Il reprend dans ses aspects principaux la longue réplique shakespearienne: "Amis, Romains, compatriotes, prêtez-moi l'oreille. Je suis venu pour ensevelir César, non pour le louer...». Le peuple exige qu'on lise le testament de César. Un des citoyens reprend la réplique du texte de Shakespeare: "C'étaient des traîtres; eux, des hommes honorables!»; cette réplique prend tout son sens en prison et apparaît ici dans toute son universalité. Antoine commence à lire le testament de César. Le peuple alors se révolte: on voit un panoramique sur la prison et on entend les cris de la révolte (comme s'il y avait une mutinerie). Ici l'ordre est donc inversé par rapport au texte où le testament est lu après la révolte. On remarque, en outre, que (comme on l'a déjà vu) on ne reprend pas l'intégralité des répliques du texte mais seulement les parties essentielles. D’autre part, la cour de la prison, avec les détenus aux fenêtres, derrière les barreaux, apparaît comme un décor extraordinaire, à la fois solennel, tragique et bien vivant.

- Le soir, dans sa cellule, Brutus raconte à ses deux codétenus, qu'il a dû fuir avec Cassius et monter une armée. Il raconte la révolte de Rome.

Acte IV (préparation de la vengeance)

- Acte IV, scène I. Arrivée d'Octave qui est interprété par un prisonnier nouvellement arrivé. On est en train de monter les décors sur la scène du théâtre. Octave - explique le metteur en scène qui fait ainsi progresser l'action - est le fils adoptif de César qui décide de s'allier à Antoine pour venger César. Il va lever une armée pour la bataille finale de Philippes. Les décors qu'on prépare sont ceux de la bataille. L'acteur interprétant Antoine a reçu une visite et il est triste.

- Acte IV, scène III (même décor). Brutus, sous la tente, attablé devant une bougie. Le vent souffle. Brutus entend la voix de César.

12 Voici le texte de Shakespeare: "Que de fois on verra le simulacre sanglant de ce César que voilà gisant sur le piédestal de Pompée, au niveau de la poussière!» 
Acte V (la bataille)

- Acte V, scène I (en plein air, probablement dans la cour de la prison). Avant la bataille, Brutus et Cassius se disent adieu. Ils sont en costume, filmés en plan taille. Le vent donne l'impression qu'ils sont sur le champ de bataille. On ne voit que le ciel en arrière-plan.

- Le jour de la «vraie» représentation, on voit le public qui pénètre dans la prison.

- Acte V, scène II. À partir d'ici le film est en couleur. Les personnages sont sur scène et chacun résume sa part de bataille, faisant ainsi suivre aux spectateurs la progression de celle-ci.

- Acte V, scène II. Suicide de Cassius.

- Acte V, scènes III et V. Cette scène reprend, en plus détaillée, celle qu'on a vue au début du film. Brutus, seul, demande à Trébonius de l'aider à mourir, il demande ensuite à un autre, puis à un autre, puis à Décius (dans le texte il ne demande pas cela aux mêmes personnages). Il demande enfin à Straton lequel lui plante une épée dans le ventre.

- On revient à la scène du début du film. Le corps de Brutus est allongé sur un drap avec une épée. Moment d'incertitude, car on ne sait pas ce qu'il va se passer, d'autant plus que tout cela n'est pas dans le texte de Shakespeare. César relève Brutus et tout le monde salue la foule qui applaudit. La trouvaille du metteur en scène a fonctionné!

- Chaque prisonnier regagne sa cellule. Ce sont les mêmes plans qu'au début. Cosimo Rega (Cassius) déclare face à la caméra: "Depuis que j'ai connu l'art, cette cellule est devenue une prison. » Il se fait ensuite un café.

- Le générique de fin informe des choses suivantes: "Cosimo Rega a publié Sumino O' Falco, Autobiographie d'un condamné à la perpétuité. Salvatore Striano (Brutus), après avoir bénéficié d'une remise de peine, est aujourd'hui acteur de théâtre et de cinéma. Giovanni Arcuri (César) a publié le livre Libre à l'intérieur.»

On le voit, les répétitions sont le moment où tous les temps se mêlent: celui des prisonniers, celui des personnages de Jules César, le temps extérieur à la prison. Il faut cependant s'attarder quelque peu sur le concept même de répétition choisi par les Taviani. La répétition est un temps mixte par excellence, celui où se confondent sans cesse la réalité et la fiction, où l'on joue la pièce, mais pas totalement. S'il l'on s'en tient au sens premier, c'est un temps répétitif, ou plutôt, faussement répétitif, car les acteurs progressent peu à peu. Il s'agit en fait du moment de l'appropriation, d'un temps incertain, non figé (contrairement à celui du spectacle) où tout semble encore permis pour les acteurs qui ne sont pas encore dans la peau de leur personnage. Et il s'agit effectivement d'un moment idéal, celui de l'entre-deux où l'on peut jouer avec la pièce, moment où les acteurs et le texte doivent chacun faire des concessions pour arriver à vivre ensemble, comme le montre ce film. Bref, c'est le temps idéal de la réécriture, de l'appropriation réciproque. 
D'autre part, le temps de la détention est la composante fondamentale qui donne une force extraordinaire ${ }^{13}$ à ce moment de réappropriation du texte de Shakespeare, la prison étant un microcosme parfait qui se prête très bien à la reconstitution de son monde. En effet, si le temps des acteurs est idéalement figé (ils sont tous condamnés à de lourdes peines), paradoxalement c'est le temps historique qui les propulse dans l'action et leur fait oublier ce temps figé. D'autre part, le temps historique devient ici extrêmement concret, il se greffe à tous leurs problèmes personnels. Comme on l'a vu, le texte shakespearien devient à sa façon la mémoire des prisonniers, ou à tout le moins il est réminiscence de choses qu'ils ont vécues. Cette rencontre entre la pièce et les détenus est donc assez extraordinaire et donne une nouvelle force à ces deux éléments; il s'agit donc bien d'une réécriture enrichissante pour Jules César.

Toute adaptation est certes une réécriture. Mais, ici, on a compris que la vraie réécriture n'est pas dans le spectacle qui sera monté (et que l'on peut voir comme une simple adaptation dont on peut penser qu'il sera assez conventionnel et qui apparaît très peu à l'écran), mais bien dans les répétitions où c'est bien une histoire différente qui se trame. Le film n'adapte donc pas la pièce, mais la reconstruit, à la fois pour le cinéma, mais aussi pour la situation particulière qu'il veut mettre en scène.

Ce n'est pas la première fois que les frères Taviani se livrent à une réécriture/ réappropriation d'une œuvre. Ils l'ont fait de façon presque classique en adaptant des nouvelles de Pirandello dans Kaos et Tu ridi. Cependant, déjà dans ces deux films, ils avaient glissé des épisodes inventés mais fidèles à l'esprit de Pirandello. Dans un autre film, Good morning Babylonia (1987), mêlant encore une fois habilement la fiction et le "réel ${ }^{14}$ », ils réécrivent l'histoire du cinéma en donnant corps à la légende (invérifiable) selon laquelle le cinéaste américain Griffith aurait été influencé par Cabiria (péplum italien de Giovanni Pastrone, 1914) pour la mise en scène des épisodes babyloniens de son Intolerance (1916). Avec César doit mourir, ils semblent renverser leur logique de réécriture et donnent l'impression, le temps de ces répétitions en prison, que c'est Shakespeare qui s'est inspiré de la vie des acteurs-détenus. On voit très bien ici, à travers l'assimilation des acteurs à leur propre rôle, à travers la manière dont les rôles débordent souvent sur des situations vraiment vécues, et qui risquent de l'être encore, le caractère universel de la pièce de Shakespeare dans un contexte tout à fait inattendu, voire presque provocateur. En fait, les détenus vivent cette histoire comme la leur, on ne sait plus s'ils réécrivent Shakespeare ou si c'est Shakespeare qui leur apprend à décoder leur propre vie. De ce fait, leurs

13 On a vu, par exemple, la puissance suggestive de la cour de la prison, augmentée par le choix du noir et blanc.

14 Je mets toujours le mot réel entre guillemets, car je ne crois pas au concept de réel. 
commentaires, et ceux des gardiens (dans d'autres scènes), sont aussi importants que l'histoire de Shakespeare.

En vérité, les Taviani suggèrent simplement qu'ils n’ont pas réécrit Shakespeare, mais que ce dernier, avec Jules César, n'a fait que réécrire le drame de la vie, toujours recommencé, plutôt qu'un simple épisode historique (et figé) de l'antiquité romaine.

\section{Adaptations cinématographiques des Taviani}

Boccace: Contes italiens (2015).

Goethe: Les Affinités électives (1996).

Ledda: Padre padrone (1977).

Pirandello: Kaos (1984); Tu ridi (1998).

Tolstoï: Le soleil même la nuit (1990); Résurrection (2001, film pour la télévision). 